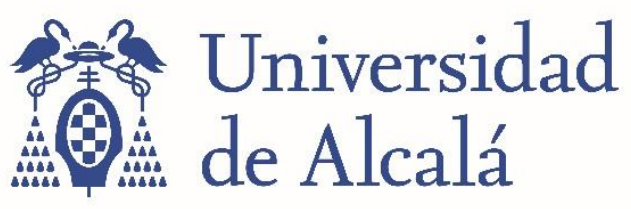

BIBLIOTECA

Document downloaded from the institutional repository of the University of Alcala: http://dspace.uah.es/

This is a postprint version of the following published document:

Angulo-Vinuesa, X., Lopez-Gil, A., Dominguez-López, A., Cruz, J.L., Andres, M.V., et al., " Simultaneous gain and phase profile determination on an interferometric BOTDA ", (September 28, 2015); Proc. SPIE 9634, 24th International Conference on Optical Fibre Sensors, 963419

Available at http://dx.doi.org/10.1117/12.2192663

Copyright 2015 Society of Photo Optical Instrumentation Engineers. One print or electronic copy may be made for personal use only. Systematic electronic or print reproduction and distribution, duplication of any material in this paper for a fee or for commercial purposes, or modification of the content of the paper are prohibited.

(Article begins on next page)

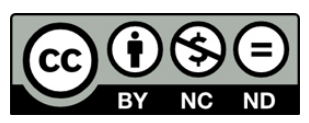

This work is licensed under a

Creative Commons Attribution-NonCommercial-NoDerivatives

4.0 International License. 


\title{
Simultaneous Gain and Phase Profile Determination on an Interferometric BOTDA
}

\author{
X. Angulo-Vinuesa*a, A. Lopez-Gil ${ }^{\mathrm{a}}$, A. Dominguez-Lopez ${ }^{\mathrm{a}}$, J. L. Cruz ${ }^{\mathrm{b}}, \mathrm{M}$. V. Andres ${ }^{\mathrm{b}}$, \\ S. Martin-Lopez ${ }^{\mathrm{a}}$, M. Gonzalez-Herraez ${ }^{\mathrm{a}}$ \\ ${ }^{a}$ Dept. de Electrónica, Universidad de Alcalá, C. Universitario, 28871, Alcalá de Henares, Spain; \\ ${ }^{\mathrm{b}}$ Dept. de Física Aplicada, ICMUV, Universidad de Valencia, 46100 Burjassot, Spain
}

\begin{abstract}
Up to now, complex (phase and intensity) measurements in Brillouin Optical Time-Domain Analysis (BOTDA) systems required complex phase modulation methods and high-bandwidth (multi-GHz) detection. In this work, we propose a novel technique that is able to retrieve simultaneously both gain/loss and phase characteristics of the Brillouin interaction by just introducing a Sagnac Interferometer (SI) on a standard BOTDA sensing scheme. The technique is described analytically and demonstrated experimentally. With this technique, a reliability increase is produced since redundant measurements can be performed.
\end{abstract}

Keywords: Brillouin Scattering, distributed optic fiber sensor, temperature sensor, strain sensor, interferometry.

\section{INTRODUCTION}

Brillouin Optical Time Domain Analysis (BOTDA) systems [1] are based on the non-linear effect known as Stimulated Brillouin Scattering (SBS) [2]. SBS manifests as a counter-propagating narrowband gain or loss when an intense and coherent light beam is introduced from one end of an optical fiber. These gain/loss profiles, known as Brillouin Gain Spectrum (BGS) or Brillouin Loss Spectrum (BLS), have a Lorentzian shape with its maximum/minimum detuned from the pump frequency by a quantity known as the Brillouin Frequency Shift (BFS - $v_{B}$ ). Associated to the amplification/attenuation curves, the BGS/BLS shows a phase profile too, called Brillouin Phase-shift Spectrum (BPS). The schematic representation of the spectral behavior of SBS waves is shown in Fig.1(a).

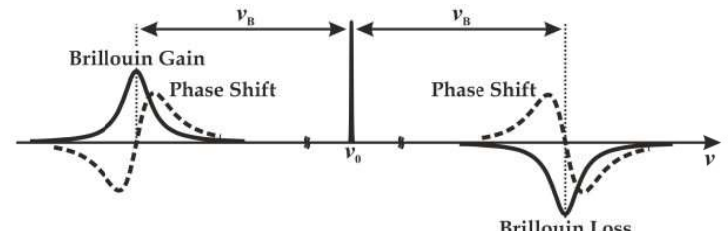

(a)

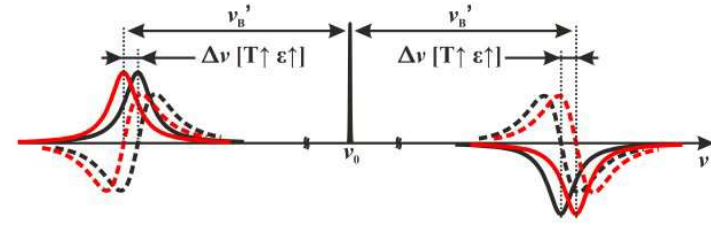

(b)

Fig. 1. (a) Schematic representation of the BGS/BLS and BPS and (b) their dependence with increased strain or temperature.

The linear dependence with strain and temperature of the BFS [3] has allowed developing Brillouin based distributed fiber sensors, where the position of the gain/loss curves has been employed to determine strain or temperature variations. Together with the amplification/attenuation profiles, the associated BPS also varies its position with any inhomogeneity in the fiber (see Fig.1(b)). Since the BPS shows a linear shape around the BFS, some systems make use of such response for sensing purposes $[4,5]$, although the complexity of the schemes (phase modulation, high bandwidth detectors, etc.) reduce their effectiveness for some applications. In this work, we propose a novel technique that is able to provide simultaneously gain/loss and phase profile responses by just introducing a Sagnac Interferometer (SI) on a standard BOTDA structure. Through the use of the SI [6], it is possible to exploit the natural non-reciprocity of the system, thus the retrieved signal intensity will be a combination of the gain or loss together with the phase difference among the waves travelling in counter-propagating directions within the SI. Through a proper filtering process and linear combinations of the retrieved waves, it is then possible to reconstruct all the four possible SBS profiles (gain, loss and respective phases). Besides from being able to retrieve the BPS with standard BOTDA components, the system is able to perform redundant temperature or strain measurements. Theoretical calculations and experimental measurements are provided to proof the effectiveness of the technique. 


\section{INTERFEROMETRIC BOTDA SYSTEM}

In standard BOTDA systems, an intensity-modulated probe wave is introduced through one side of the fiber, opposite to the pump wave, and is amplified or attenuated if its frequency detuning from the pump matches the BFS of the tested fiber (Fig.2(a)). As it can be seen, in this procedure the fiber loop structure (both ends are employed to input signals) is not fully exploited, as the measured probe wave is measured directly. By introducing a SI, the probe wave is divided in two (Probe 1 and Probe 2), travelling in opposite directions within the fiber, thus interfering with itself before being detected either in transmission or reflection (Fig.2(b)). The SBS created by the counter-propagating pump affects only Probe 2 (SBS is strictly non-reciprocal), and hence is visualized at the output of the SI after interference with Probe 1.

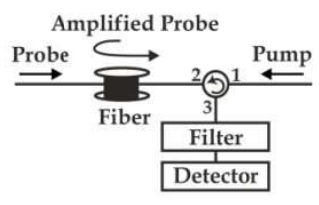

(a)

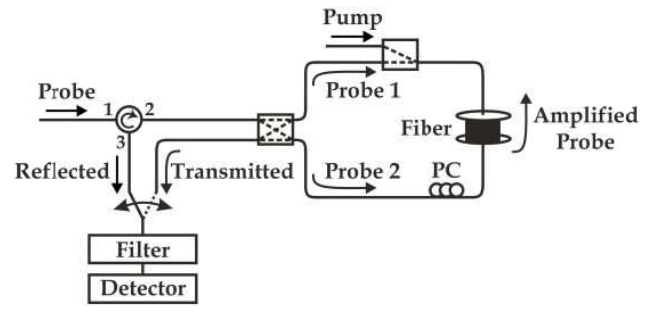

(b)

Fig. 2. (a) Standard BOTDA scheme and (b) the proposed variation with a Sagnac Interferometer within the tested fiber.

Unlike in conventional SI schemes (where polarization alignment is searched), in this case, a polarization misalignment is deliberately produced through the Polarization Controller (PC) to ensure a phase mismatch among Probes 1 and 2. Experimentally, the polarization is tuned so both output intensities (reflected and transmitted) are similar. The pump wave will only interact with one of the probe waves (Probe 2), and Probe 1 will act as a reference signal. Under such conditions, the upper and lower frequency sidebands of the probe will be amplified/attenuated the same amount, although both will suffer the same nonlinear phase shift. Mathematically, if normalized fields are assumed for simplicity purposes, the AC component of the transmitted and reflected gain/loss intensities can be expressed as:

$$
\begin{array}{ll}
|E t G|^{2}=\left|e^{-j \phi}-G e^{+j \phi}\right|^{2} & |E t L|^{2}=\left|e^{-j \phi}-L e^{+j \phi}\right|^{2} \\
|E r G|^{2}=\left|e^{-j \phi}+G e^{+j \phi}\right|^{2} & |E r L|^{2}=\left|e^{-j \phi}+L e^{+j \phi}\right|^{2}
\end{array}
$$

where $\phi$ is the relative phase delay among the counter-propagating waves, and $G$ and $L$ can be approximated to:

$$
G=e^{\left(g_{B} P_{p} \Delta z\right)} \approx 1+g_{B}(v) P_{p} \Delta z \quad L=e^{\left(-g_{B} P_{p} \Delta z\right)} \approx 1-g_{B}(v) P_{p} \Delta z
$$

being $P_{p}$ the pump power, $\Delta z$ the spatial resolution and $g_{B}(v)$ the complex Brillouin gain/loss coefficient:

$$
g_{B}(v)=g(v)+j \sigma(v)=\frac{g_{p} / A_{e f f}}{1+j\left(\frac{v-v_{B}}{\Delta v_{B}}\right)}
$$

where $g_{p}$ is the Brillouin gain factor, $A_{\text {eff }}$ the effective area, $v$ the optical frequency shift, $v_{B}$ the BFS and $\Delta v_{B}$ the Brillouin gain bandwidth. Equations in (1) can now be expressed analytically as:

$$
\left|E_{r}^{t} G\right|^{2} \propto g \pm \sigma(v) \sin (2 \phi) \quad\left|E_{r}^{t} L\right|^{2} \propto-g \pm \sigma(v) \sin (2 \phi)
$$

It can be clearly seen their proportionality to the gain/loss $(g)$ and the phase response of the SBS $(\sigma)$ as long as a phase delay is present between the two propagation directions $(\phi \neq 0)$.

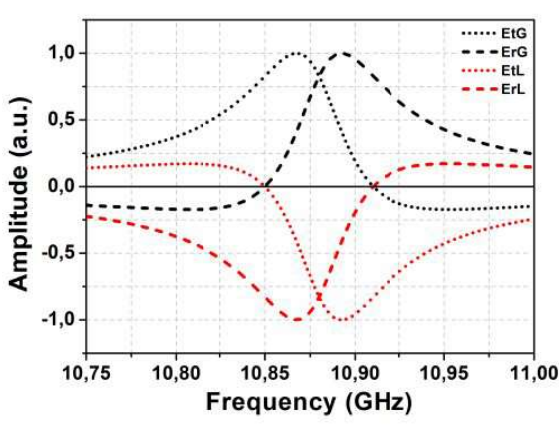

(a)

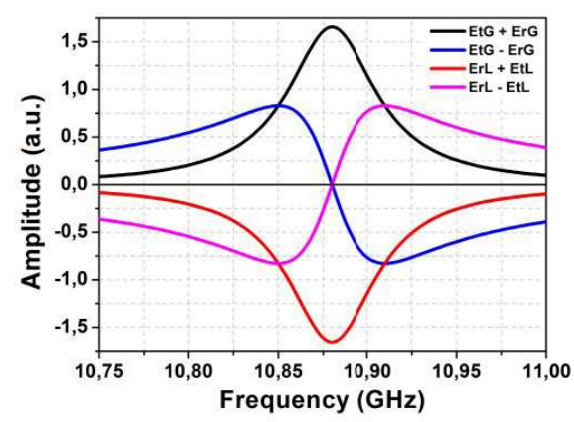

(b)

Fig. 3. (a) Transmitted and reflected gain and attenuation curves ( $E t G, E r G, E t L$ and $E r L$ ) and (b) linear combinations $(E t G \pm E r G$ and $E r L \pm E t L)$ that determine the pure gain/loss profiles and their respective phase-shifts. 
Fig.3(a) shows the expected response for the gain and loss sidebands, both in transmission and reflection. It can be seen that each of the obtained independent curves (EtG, ErG, EtL and $E r L$ ) do not match the expected typical Lorentzian shape. However, if the retrieved profiles are added and subtracted respectively (EtG $\pm E r G$ or $E r L \pm E t L)$, Fig.3(b), pure amplification/attenuation shapes and their corresponding matching phases are retrieved. Thus, through the use of an interferometric BOTDA system and proper filtering, it is possible to retrieve simultaneously either the gain or loss Brillouin profile and the respective phase-shift characteristic. In the following section, we will demonstrate the effectiveness of the technique applied on a real temperature sensor.

\section{EXPERIMENTAL SETUP AND RESULTS}

The developed experimental setup is based on a standard BOTDA system although, in this case, the Fiber Under Test (FUT) is inserted into a Sagnac loop. The schematic diagram of the system can be seen in Fig.4.

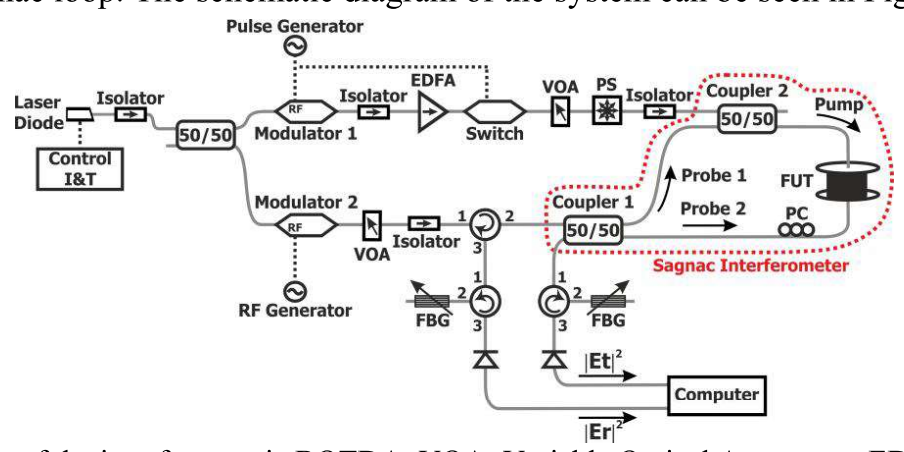

Fig. 4. Schematic drawing of the interferometric BOTDA. VOA: Variable Optical Attenuator; EDFA: Erbium Doped Fiber Amplifier; PS: Polarization Scrambler; FBG: Fiber Bragg Grating; PC: Polarization Controller; FUT: Fiber Under Test.

The probe wave is obtained through an intensity modulation of the master laser (Modulator 2 in the image) yielding a Double Side-Band (DSB) modulation with no carrier. $\sim 1 \mathrm{~mW}$ probe sidebands are launched into the SI through Coupler 1. The $25 \mathrm{~ns}$ pump pulses are shaped using Modulator 1 and a high-speed optical switch (to achieve high extinction ratio). Upon entrance into the SI through Coupler 2, the pulses have a peak power of $\sim 60 \mathrm{~mW}$. As stated before, only Probe 2 will experience the SBS gain/loss process; the lower frequency band will be amplified while the upper one attenuated, both of them suffering the same nonlinear phase shift. A certain polarization misalignment is introduced in the PC so that a certain non-reciprocal phase shift will arise between the two paths (clockwise and counter-clockwise). In this way, the observed interference will be a combination of the gain/loss profile and the phase shift. At the SI output, each of the desired transmitted or reflected waves (EtG and $E r G$ or $E t L$ and $E r L)$ are obtained through a narrow ( 33 pm) tunable Fiber Bragg Gratings (FBG) and detected on independent optical detectors so the retrieved information can be then properly treated.
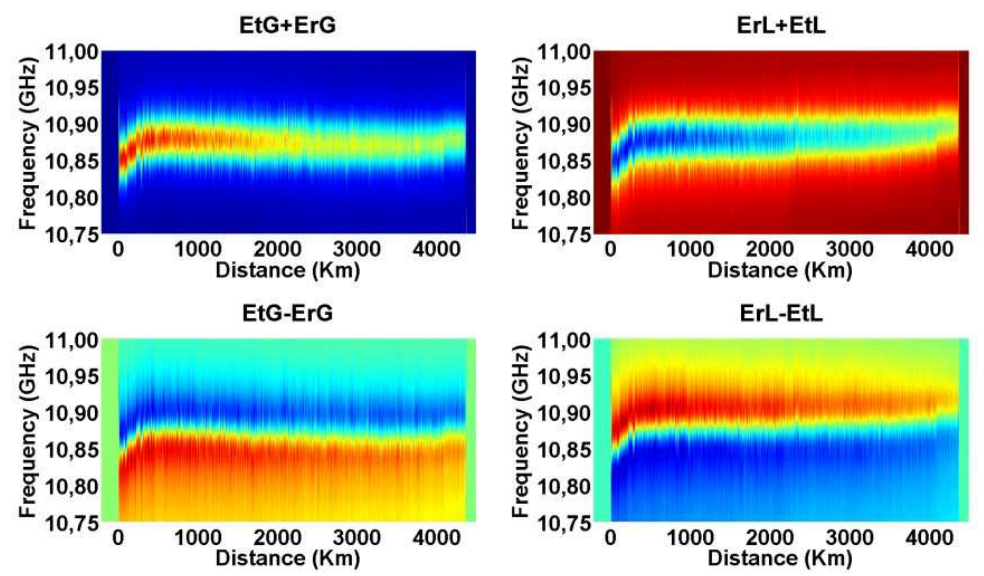

Fig. 5. Full frequency sweep for linear combinations among the retrieved intensity waves; $E t G \pm E r G$ and $E r L \pm E t L$.

In order to confirm the performance of the system as a sensor, a $4.3 \mathrm{~km}$ long fiber (composed of two spools of $4 \mathrm{~km}$ and $300 \mathrm{~m}$ ) with a maximum BFS of $\sim 10.88 \mathrm{GHz}$ (at $1550 \mathrm{~nm}$ and $25^{\circ} \mathrm{C}$ ) was tested. At the very far end of the fiber $(4.3$ $\mathrm{km}), 2.5$ meters of fiber where heated up to $83^{\circ} \mathrm{C}\left(\sim 58^{\circ} \mathrm{C}\right.$ increase). The probe wave was swept from $10.75 \mathrm{GHz}$ until 
$11.10 \mathrm{GHz}$, and the transmitted and reflected gain and loss signals were obtained with 1024 averages. Fig.5 shows the full 3D maps of the obtained linear combinations, where it is shown the expected gain/loss and phase shapes. If the far end of the fiber is zoomed (Fig.6), and compared with a non-heated region, it is clear the frequency increase of $75 \mathrm{MHz}$, which perfectly matches the expected temperature-induced shift for a fiber with $1.3 \mathrm{MHz} /{ }^{\circ} \mathrm{C}$ sensitivity $\left(+58^{\circ} \mathrm{C}\right)$.

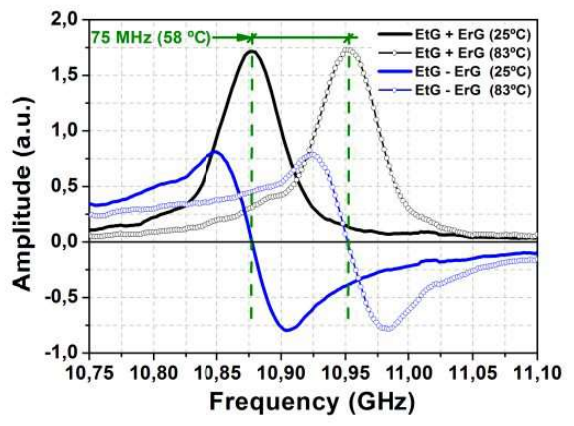

(a)

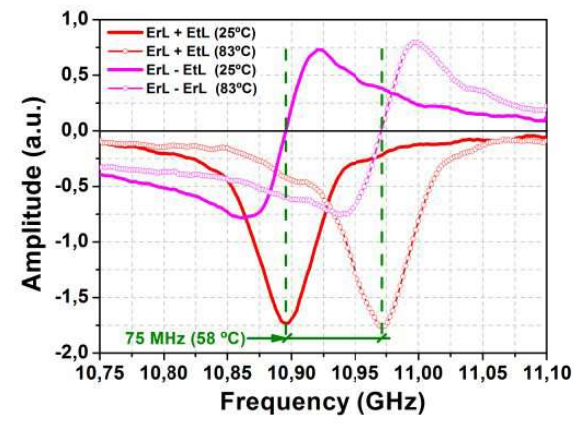

(b)

Fig. 6. Experimental results for fiber regions with normal temperature $\left(25^{\circ} \mathrm{C}\right)$ and a heated region at the far end $\left(83^{\circ} \mathrm{C}\right)$.

(a) Gain and (b) loss response with their respective phase profiles.

As we can see, the performance of the experimental setup confirms the effectiveness of the proposed technique. The possibility of being able to obtain simultaneously gain or loss profiles and their respective phase responses provides the advantage of redundant measurements, which increases the effectiveness of the technique in terms of sensing accuracy.

\section{CONCLUSIONS}

In summary, we have demonstrated a novel technique that, through the introduction of a Sagnac Interferometer (SI), is able to exploit the non-reciprocal feature of a BOTDA. When properly set, the setup produces signals that are the combination among gain/loss and Brillouin-induced phase shift. Using linear combinations among the retrieved signals, it is possible to reconstruct simultaneously the BGS and BPS profiles. This provides redundant measurements, increasing the efficiency of the system. Through this technique, it is possible to retrieve the phase profile with standard BOTDA components, instead of complex phase modulation schemes. This opens the gate to exploit the linear shape of the phase profile in the BFS region for more effective inhomogeneity determinations, even with current equipment.

\section{ACKNOWLEDGEMENTS}

This work was supported by the ERC through Starting Grant U-FINE (grant 307441), the Spanish "Plan Nacional de I+D+i" through projects TEC2012-37958-C02-01/02 and TEC2013-45265-R, the INTERREG SUDOE program ECOAL-MGT and the Comunidad de Madrid under projects EDISON and SINFOTON. S. Martin- Lopez acknowledges support from the Spanish "Ministerio de Ciencia e Innovación" through a "Ramón y Cajal" contract. J. L. Cruz and M. V. Andrés acknowledge financial support from the Spanish "Ministerio de Economía y Competitividad" and FEDER funds (project TEC2013-46643-C2-1-R), and the Generalitat Valenciana (project PROMETEOII/2014/072).

\section{REFERENCES}

[1] Horiguchi, T., and Tateda, M., "BOTDA - Nondestructive measurement of single-mode optical fiber attenuation characteristics using Brillouin interaction: Theory," IEEE J. Lightwave Technol. 7(8), 1170-1176 (1989).

[2] Agrawal, G. P., [Nonlinear fiber optics - 4th Edition], Academic Press, San Diego, 9 (2007).

[3] Niklés, M., Thévenaz, L., and Robert, P. A., "Brillouin gain spectrum characterization in single-mode optical fibers," IEEE J. Lightwave Technol. 15(10), 1842-1851 (1997).

[4] Zornoza, A. Sagües, M., and Loayssa, A., "Self-heterodyne detection for SNR improvement and distributed phase-shift measurements in BOTDA,” IEEE J. Lightwave Technol. 30(8), 1066-1072 (2012).

[5] Dossou, M., Bacquet, D., and Szriftgiser, P., "Vector Brillouin optical time-domain analyzer for high-order acoustic modes," Opt. Letters 35(22), 3850-3852 (2010).

[6] Lee, B. H., Kim, Y. H., Park, K. S., Eom, J. B., Kim, M. J., Rho, B. S., and Choi, H. Y., "Interferometric fiber optic sensors," Sensors 12(3), 2467-2486 (2012).

*xabier.angulo@uah.es; phone+34-91-885-69-14 